\title{
¿POR Qué Heidegger lleva A CABO UNA REHABILITACIÓN DE LA PHRÓNESIS ARISTOTÉLICA?
}

\author{
Ángel Xolocotzi Yáñez \\ Benemérita Universidad Autónoma de Puebla \\ angel.xolocotzi@gmail.com
}

$\mathrm{Al}$ amigo Franco Volpi
In memoriam

\begin{abstract}
For three decades Franco Volpi's thesis concerning the Aristotelianism of Heidegger has been grounds for investigation. Nevertheless, it has remained pending until now the task of delving deeply into the motives for which Heidegger would measure his strength against the great philosopher Aristotle. This paper is a contribution to this line of investigation into the preoccupations of the young Heidegger in his first lessons in Freiburg, especially in the post-war semester of 1919, as well as the discussions he sustained with the radical philosophies of Dilthey, Rickert, and Husserl. This will allow us to understand better why Heidegger was required to rehabilitate the practical philosophy of Aristotle. Key words: Phronesis, practical philosophy, pre-theoretic sphere, circumspection, Heideggerian appropriation of Aristotle.
\end{abstract}

\section{Resumen}

Desde hace tres décadas la tesis de Franco Volpi en torno al aristotelismo de Heidegger ha impulsado la investigación. Sin embargo hasta el momento había quedado pendiente profundizar en los motivos que hicieron de Aristóteles el gigante filosófico con el cual Heidegger medirá sus fuerzas. El presente trabajo es una contribución en esa línea al tematizar las inquietudes del joven Heidegger en sus primeras lecciones en Friburgo, especialmente en el semestre de posguerra de 1919, así como las discusiones que sostenía con las filosofías radicales de Dilthey, Rickert y Husserl. A partir de eso se entenderá de mejor forma porqué Heidegger requirió rehabilitar la filosofía práctica de Aristóteles.

Palabras clave: Phrónesis, filosofía práctica, esfera preteorética, circunspección, apropiación heideggeriana de Aristóteles.

*Recibido: 10-05-09. Aceptado: 02-11-09. 


\section{La necesidad del replanteamiento teorético del conoci- miento}

El papel determinante de Aristóteles en la propuesta ontológica heideggeriana ha pasado a ser una constante en las interpretaciones contemporáneas. La abrumadora bibliografía secundaria ha abarcado prácticamente todas las aristas de los impulsos que Heidegger recibió de Aristóteles, especialmente en su camino a Ser y tiempo. Sin embargo, todavía hay ámbitos que merecen una atención especial en aras de llevar a cabo una comprensión más precisa de los motivos que condujeron al filósofo de Meßkirch a dedicarse de esa manera tan intensa a la obra del Estagirita. Con ello también se contribuye a esclarecer el modo de acercamiento $\mathrm{y}$ los fines que tuvieron tales lecturas.

Mucho se ha escrito ya sobre la rehabilitación de la filosofía práctica que Heidegger llevó a cabo a partir de su interpretación aristotélica, especialmente su apropiación de la phrónesis. No obstante, un aspecto que no ha sido suficientemente destacado en la literatura secundaria concierne al tránsito de la crítica radical a la dimensión teorética, iniciada por Heidegger de modo concreto en 1919, y sus interpretaciones fenomenológicas de la phrónesis aristotélica en 1921. En este sentido, el trabajo que aquí presento buscará esclarecer la relación entre los intereses temáticos en los que estaba inmerso Heidegger, después de su tesis de habilitación, y su posterior aproximación a Aristóteles ${ }^{1}$. Más allá de apegarnos a la propia interpretación de Heidegger en torno a la dirección que adquirió su pensar, ${ }^{2}$ tomaré como hilo conductor la pregunta

${ }^{1}$ A pesar de que en 1916 Heidegger había comunicado a su novia Elfride la intención de sostener un curso sobre Aristóteles y la escolástica, no hay evidencia de que éste haya tenido lugar. En los Quästurakten del Universitätsarchiv Freiburg (B 17/61) consta que en el semestre de invierno de 1916/17 la lección de Heidegger fue "Preguntas fundamentales de la lógica." De esta manera, las lecciones en donde Heidegger interpreta a Aristóteles tendrán lugar a partir de 1921. Cf. Martin Heidegger: ¡Alma mía! Cartas a su mujer Elfride 1915-1970, Buenos Aires: Manantial 2008, p. 60.

${ }^{2}$ En varias ocasiones Heidegger interpreta la relación entre la pregunta por el ser, pregunta guía de su pensar, y los impulsos recibidos a partir de la tesis doctoral de Franz Brentano sobre Aristóteles. Al respecto puede verse su lección inaugural de entrada a la Academia de las Ciencia de Heidelberg así como la carta de respuesta al jesuita Richard- 
siguiente: ¿Por qué Heidegger lleva a cabo una rehabilitación de la phrónesis aristotélica?

Para ello me centraré inicialmente en la problemática en la que se inserta Heidegger a partir de su joven experiencia docente, principalmente en el entramado conformado por las propuestas filosóficas de Wilhelm Dilthey, Heinrich Rickert y Edmund Husserl. Sabemos que una primera aproximación a lo desplegado en estas propuestas enfatizó la posibilidad de esclarecimiento del conocimiento más allá de una mera tematización teorética que hundía sus raíces en la modernidad. Ya desde 1917, Heideggger había detectado esto: "Este invierno he dicho que era absurdo establecer los límites del conocimiento —escribe a su esposa Elfride-; más bien, el conocimiento debe ser llevado a la mayor profundidad posible"3.

Como veremos, "llevar el conocimiento a la mayor profundidad posible” exigirá la remisión a propuestas que cuestionen un carácter teorético apegado a la modernidad y abran dimensiones abarcadoras de la theoría desde una perspectiva radical. En su lección del semestre de verano de 1922 en torno a Aristóteles así lo indicará Heidegger:

En la modernidad el conocer fue a parar a otro campo de interés completamente diferente: límites, validez, posibilidades y criterios del aseguramiento; a partir de ahí [el conocer] tomó un nuevo sentido en la vida, arrastrando consigo en la estructura del problema, etc. a la tradición anterior, no debilitada ${ }^{4}$.

Esta crítica al conocimiento heredado en esquemas de la modernidad encuentra, para el joven Heidegger, alternativas viables en la interpretación

son y su escrito Mi camino en la fenomenología. Véase también: Ángel XoLOCOTZI: “Mi Brentano es el de Aristóteles'. La presencia metódica y temática de Franz Brentano en la ontología fundamental de Martin Heidegger" en Á. XoLOCOTZI (coord.): Actualidad de Franz Brentano, México: UIA 2006.

${ }^{3}$ Heidegger: jAlma mía!, p. 74.

4 Martin Heidegger: Phänomenologische Interpretationen ausgewählter $A b$ handlungen des Aristoteles zur Ontologie und Logik, GA 62, Frankfurt am Main: Klostermann 2005, pp. 119-s. 
novedosa de la phrónesis aristotélica. De esa forma, como veremos, los impulsos dados por contemporáneos como Dilthey o Rickert encuentran en Aristóteles la confirmación del ámbito radical buscado.

\section{E1 descubrimiento de la intuición hermenéutica}

Desde hace más de treinta años Franco Volpi arriesgó la tesis de que la radicalidad de la filosofía de Heidegger dependía en gran medida de su lectura de Aristóteles ${ }^{5}$. A pesar de que era evidente que antiguos alumnos de Heidegger como Hans-Georg Gadamer, Hannah Arendt, Hans Jonas, Leo Strauss o Joachim Ritter concretaban de cierta forma la rehabilitación de la filosofía práctica con base en Aristóteles, todavía parecía atrevido para algunos, como Heinrich Rombach, Max Müller o Rudolf Berlinger, derivar la radicalidad heideggeriana en cierta apropiación de Aristóteles. Sin embargo, la publicación de las primeras lecciones de Heidegger a partir de 1985 ha dado en parte la razón a Volpi ${ }^{6}$. Actualmente contamos con suficientes trabajos que muestran la importancia de Aristóteles y especialmente de la Ética Nicomáquea en el despliegue de aspectos centrales de Ser y tiempo ${ }^{7}$. De ese modo, el trabajo pionero de Volpi que insistió en el puente entre Aristóteles y Heidegger queda suficientemente confirmado ${ }^{8}$.

Siguiendo la metáfora anatómica propuesta por Heidegger en 1923, esto es, que Aristóteles constituyó su "modelo" (Vorbild) mientras que

${ }^{5}$ Ya desde 1976, fecha en que Franco Volpi publica su tesis doctoral sobre Heidegger y Brentano, se hallaba la corazonada de que Heidegger era más aristotélico de lo que se pensaba. Cf. Ángel Xolocotzi: "Perspectivas de la filosofía y cuidado de la tradición. Diálogo con Franco Volpi”, Revista de Filosofía (Universidad Iberoamericana), 112 (enero-abril 2005), pp. 31-45.

${ }^{6}$ Aunque en 1985 se publica la primera lección de Heidegger como Privatdozent en Friburgo, no será sino hasta 2005, con la publicación del vol. 62 de la Gesamtausgabe, cuando queden bien documentadas las aseveraciones que Volpi había insinuado desde 1976.

${ }^{7}$ Por ejemplo así lo señalan importantes trabajos como los de Ted SADLER: Heidegger and Aristotle. The question of Being, London: Athlone 1996 y Chiara AgNELLO: Heidegger e Aristotele. Verità e linguaggio, Genova: Il melangolo 2006.

${ }^{8}$ Para esto cf. Franco VOLPI: "Sein und Zeit. Homologien zur Nikomachischen Ethik", Philosophsiches Jahrbuch 96 (1989), pp. 225-240. 
Husserl fue quien le "puso los ojos" (die Augen hat mir Husserl eingesetzt), podríamos añadir que esta colocación de "ojos fenomenológicos" por parte de Husserl ocurrió, empero, en un rostro ya afectado por la inconformidad con el tratamiento que se daba al ámbito cognoscitivo. Así, el método fenomenológico del que se apropió Heidegger fue instaurado en un terreno preocupado por la esfera previa a aquella en la cual se llevan a cabo las ciencias. Se trataba de pensar un ámbito que escapara a la causalidad de las ciencias y a su respectiva aprehensión teórico-cognoscitiva. Sabemos que esta posibilidad fue tematizada por Dilthey al pensar el ámbito de la comprensión de las ciencias del espíritu y por los neokantianos de Baden, especialmente por Rickert, al pensar el ámbito trascendental del valor. Tanto la comprensión diltheyana como la valoración rickertiana apuntaban a un ámbito subjetivo que se escapaba a toda explicación causal. Si la explicación causal de las ciencias naturales dependía fundamentalmente de las posibilidades abiertas por el dominio teorético de la representación moderna, entonces la posibilidad de hablar de un conocimiento no causal explicativo, sino más bien comprensivo, exigía el rompimiento con la representación moderna y la apertura de otros modos de conocer

De esta manera, Heidegger descubre en la autognosis de la comprensión de la vida en Dilthey y en la toma de posición valorativa en torno a los juicios en Rickert posibilidades de tematización que rompían de alguna forma con la tradición teorética en la que se movía tanto el positivismo como algunas líneas apegadas a la herencia moderna ${ }^{10}$. Frente al dominio de la representación y su acceso teórico-cognoscitivo se abre la posibilidad de un conocimiento práctico. Dilthey buscará a lo largo de su obra concretar su proyecto de una crítica de la razón histórica,

${ }^{9}$ Cf. Martin Heidegger: Ontología. Hermenéutica de la facticidad, trad. Jaime Aspiunza, Madrid: Alianza 1999, p. 22 (alemán: Ontologie (Hermeneutik der Faktizität), GA 63, Frankfurt am Main: Klostermann 1982, p. 5): "Mentor en la busqueda fue el Lutero joven; modelo, Aristóteles, a quien aquél odiaba. Impulsos me los dio Kierkegaard, y los ojos me los puso Husserl."

${ }^{10}$ Esto ha sido trabajado con detalle en Ángel XoLOcoTzI: Subjetividad radical y comprensión afectiva. El rompimiento de la representación en Rickert, Dilthey, Husserl y Heidegger, México: Plaza y Valdés 2007. 
mientras que Rickert ubicará al conocimiento más bien en el ámbito de la razón práctica.

Las posibilidades radicales de un tratamiento de la subjetividad tal como Heidegger las ve en Dilthey y Rickert se verán transformadas mediante la intervención fenomenológica. Que Heidegger vea con ojos fenomenológicos la posibilidad de una filosofía práctica eso ya lo había notado Husserl, así lo señala en 1922 en una carta a Natorp:

Su forma de ver, de trabajar fenomenológicamente y el campo mismo de sus intereses — nada de eso está tomado simplemente de mí, sino que [está] arraigado en su propia originalidad [...] Él habla sobre aquello que la investigación profunda prospectiva y fenomenológica de las ciencias del espíritu le enseña $[\ldots]^{11}$.

Aunque Heidegger había entrado en contacto epistolar con Husserl en 1914, cuando éste todavía era profesor en Gotinga, y Husserl haya llegado a Friburgo en 1916, será en 1919 cuando Heidegger inicie sus labores docentes en el marco de la cátedra husserliana ${ }^{12}$. Tal inicio docente expone sin embargo confrontaciones en las que se había insertado el joven Heidegger por lo menos desde 1917. Se trataba de la confrontación entre la problemática de las ciencias del espíritu, discutida por su maestro neokantiano Heinrich Rickert, y la fenomenología de su protector Edmund Husserl ${ }^{13}$. De esta forma la lectura con "ojos fenomenológicos" de los textos de Rickert conducirá a un punto de partida determinan-

${ }^{11}$ Edmund Husserl: Briefwechsel, volumen V: Die Neukantianer, Dordrecht: Kluwer 1994, p. 150. Se trata de la carta del 1 de febrero de 1922.

${ }^{12}$ Por la carta del 3 de julio de 1914 de Heidegger a su maestro Rickert sabemos que el primero había entrado en contacto epistolar con Husserl desde ese mismo año. Cf. Martin Heidegger y Heinrich Rickert: Briefe 1912-1933, Frankfurt am Main: Klostermann 2002, p. 19. Sin embargo Heidegger iniciará en 1916, poco después de su habilitación, su camino académico como Privatdozent en la Cátedra II de Filosofía. Será a principios de 1919 cuando labore con esa categoría académica en la Cátedra I bajo el regazo de Edmund Husserl.

${ }^{13}$ Para documentar esto cf. las cartas de Heidegger a su esposa Elfride y al medievalista Grabmann: HeIdegGer: ¡Alma mía!, p. 75; Hermann KÖstLER: "Heidegger schreibt an Grabmann", Philosophisches Jahrbuch 87 (1980), p. 74. 
te para el camino filosófico de Heidegger, así lo señala en una carta a Rickert del 27 de enero de 1920:

Más aún, al penetrar la pregunta por la estructura de la intuición fenomenológica de las vivencias puras encontré que el concepto de "interpretación de sentido" conduce al centro, que la intuición fenomenológica no es un contemplar las vivencias como cosas, sino que la referencia, que corresponde a la vivencia, entre el sentido de ejecución y el sentido de contenido exige una forma genuina y adecuada del intuir, que yo introduzco como intuición comprendedora, hermenéutica ${ }^{14}$.

El punto determinante para Heidegger ya un año antes de la redacción de esta carta será precisamente la propuesta de una intuición fenomenológica que no contemple vivencias y cosas, sino que más bien aprehenda el vivenciar y lo vivenciado desde otro punto de partida. Eso será como él mismo señala la propuesta de una intuición fenomenológica comprendedora.

La lección de posguerra en 1919 (Kriegsnotsemester) toma así como hilo conductor el siguiente móvil: "nos movemos en la aridez del desierto con la esperanza de comprender intuitivamente y de intuir comprensivamente en lugar de conocer siempre cosas [...]"15. La posibilidad de una intuición comprendedora se enfrenta pues a la hegemonía de intuiciones cognoscitivas que Heidegger agrupará bajo la bandera de lo teorético. De ese modo, el programa heideggeriano inicial exige una reubicación del privilegio otorgado a lo teorético:

Se ha de romper con esta primacía de lo teorético, pero no con el propósito de proclamar un primado de lo práctico o de introducir otro elemento que muestre los problemas

${ }^{14}$ Heidegger y Rickert: Briefe, pp. 47-s. (cursivas mías).

15 Heidegger: La idea de la filosofía y el problema de la concepción del mundo, trad. Jesús Adrián Escudero, Barcelona: Herder 2005, p. 79 (alemán: "Die Idee der Philosophie und das Weltanschaunngsproblem", Zur Bestimmung der Philosophie, GA 56/57, Frankfurt am Main: Klostermann 1987). 
desde una nueva perspectiva, sino porque lo teorético mismo y en cuanto tal remite a algo pre-teorético ${ }^{16}$.

Tal remisión de lo teorético a lo preteorético lo desplegará Heidegger en el análisis de la vivencia del entorno. Ésta no es una vivencia peculiar, sino que "nuestras vivencias se enmarcan con frecuencia y en la mayoría de los casos en un entorno" por ello "el vivir del entorno no es una contingencia, sino que radica en la esencia de la vida en y para sí” y por eso - agregará Heidegger_ “ "sólo en ocasiones excepcionales estamos instalados en una actitud teórica" $"$.

En la vivencia del mundo circundante o entorno no vemos, intuimos, cosas aisladas que después se inserten en una totalidad constituyente de mundo, sino que más bien la vida mundea, es decir se encuentra ya rodeada de significados del entorno. Se trata de que aquí lo significativo sea develado como lo primario, "sin ningún rodeo intelectual que pase por la captación de una cosa"18. Este carácter primario de lo circundante desde donde puede abstraerse algo para ser conocido como cosa es el ámbito preteorético que Heidegger busca destacar como punto de partida.

De ese modo, la crítica a lo teorético descansa en el hecho de que éste está determinado a partir de lo cósico del mundo, aprehendido cognoscitivamente. La posibilidad del ámbito preteorético abre entonces la tematización de lo entornado en cuanto significativo y de su correspondiente aprehensión comprendedora. De esta forma Heidegger se atreve a vislumbrar una intuición que no se apegue al carácter cognoscitivo de lo teorético, sino más bien al carácter hermenéutico de lo preteorético.

Así como Husserl desplegó su propuesta fenomenológica cuestionando la ingenuidad de la aprehensión independiente de las cosas y remitiendo necesariamente al ámbito vivencial, así Heidegger avanzará fenomenológicamente al remitir al ámbito de las vivencias, pero ahora no en términos teoréticos, sino hermenéuticos.

\footnotetext{
${ }^{16}$ HeidegGer: La idea de la filosofía, pp. 70-s.

${ }^{17}$ Heidegger: La idea de la filosofía, p. 106.

${ }^{18}$ Heidegger: La idea de la filosofía, p. 88.
} 
No es difícil detectar que el punto de partida certero de Ser y tiempo en 1927, es decir, el análisis del mundo circundante, lleva a cabo la concreción de lo iniciado ya en 1919 a partir de esta transformación de la intuición en tanto conocimiento a la intuición como comprensión.

Sin embargo, a pesar de que esta línea de trabajo se insertaba metódicamente en la visión fenomenológica y temáticamente en la crítica al predominio teorético en el marco de la discusión sobre la legitimidad de las ciencias del espíritu, Heidegger descubre que la posibilidad de una filosofía no-teorética no era asunto propio de la filosofía contemporánea, sino que más bien la hegemonía de lo teorético remitía a un olvido de la legitimidad de lo preteorético. Por ello se hará necesaria una remisión a la tradición, tal como lo señala en una carta de principios de 1922: "He alcanzado una gran seguridad, en verdad ya no tengo nada que aprender de los filósofos contemporáneos; me resta sólo medir mis fuerzas con los que juzgo los filósofos más decisivos de la historia" ${ }^{\text {"19 }}$.

Sobra decir que el filósofo decisivo con el que Heidegger medirá sus fuerzas es Aristóteles. Incluso él mismo reconocerá muchos años más tarde en confesión a su alumno Gadamer lo determinante de su confrontación con Aristóteles: "Aunque los años de Marburgo hayan sido determinantes para la elaboración de Ser y tiempo, la genuina pregunta por el ser la traje ya de mis varios años de confrontaciones con Aristóteles en la primera época en la universidad [de Friburgo]"20.

A pesar de que Heidegger indica su medición de fuerzas con Aristóteles a principios de 1922, la aventura ya había comenzado un semestre antes, en el semestre de verano de 1921 con la lectura de De anima y con la asistencia de Hans Jonas, Max Horkheimer, Karl Löwith, Günther Stern (Anders), Hans Reiner y Oskar Becker, entre otros ${ }^{21}$.

${ }^{19}$ Heidegger: Mein liebes Seelchen!, p. 133. Se trata de una carta a su esposa Elfride del 26 de enero de 1922.

${ }^{20}$ Ausgewählte Briefe Martin Heidegger an Hans-Georg Gadamer, Jahresgabe der Martin-Heidegger-Gesellschaft 2005/2006, p. 38. Se trata de la carta del 3 de septiembre de 1960.

${ }^{21}$ Esta información fue extraída de los Quästurakten del Universitätsarchiv Freiburg: B 17/71. De acuerdo con ello, Heidegger sostuvo en el semestre estival de 1921 
Sabemos que estas interpretaciones se prolongarán tanto en Friburgo como en Marburgo y asistirán aquellos que posteriormente, por caminos propios, llevarán a cabo una rehabilitación de la filosofía práctica: Hans-Georg Gadamer, Hans Jonas, Hannah Arendt, Leo Strauss y Joachim Ritter.

Pero ¿cómo se lleva a cabo la medición de fuerzas con Aristóteles a partir de la necesidad de un ámbito preteorético? Veamos esto.

\section{E1 carácter circunspectivo de la phrónesis}

Con ojos fenomenológicos Heidegger mantiene fehacientemente que el método no es simplemente un medio técnico, el cual pudiese ser aplicado indiferentemente a cualquier objeto. Más bien, el método se determina a partir del objeto. Esto lo lleva entonces a sostener que la vida misma en su entorno debe determinar su modo de acceso. En otras palabras, la vida es descubierta en un modo acorde con ella misma. El estar descubierto de la vida nos remite a una determinada forma en la cual la vida es verdadera. Esa determinada forma en que la vida es accesible, descubierta, es lo que Heidegger advierte inicialmente en su lectura de Aristóteles como una forma del aletheúein, del ser verdad.

Aristóteles vio acertadamente que hay diversos modos del aletheúein, es decir, diversos modos en los que el ente es descubierto. El hacer accesible la vida filosóficamente, es decir, su desencubrimiento, ocurre como un modo particular del aletheúein. Ya que Aristóteles mantiene a la vista que los modos del aletheúein dependen del objeto, entonces el modo propio del aletheúein de la vida sólo es posible a partir de la determinación de su ser. Pero la vida o zoé para Aristóteles tiene su ser en el movimiento o kínesis. Por ello el hacer accesible la vida tiene que ver con el ser cinético de ésta ${ }^{22}$.

la lección "San Agustín y el Neoplatonismo" que contó con 67 alumnos inscritos y el seminario "De anima" con 74 participantes.

${ }^{22}$ En la lección sobre Aristóteles del semestre de verano de 1922 Heidegger destacará que todos los fenómenos tematizados a partir de Aristóteles son vistos a partir del "carácter fundamental del movimiento". Por ello más adelante indica que "[...] con la interpretación de este fenómeno [kínesis] se obtiene una característica categorial del 
El tender cinético a hacer visible o descubrir, Aristóteles lo ubica en la esencia del ser humano: pántes ánthropoi toû eidénai orégontai phýsei $^{23}$. El eidénai significa un "saber" que de acuerdo con Heidegger debería ser entendido como un "ver más" o un "más a la vista": málista eidénai ${ }^{24}$. El más de la visión significaría la búsqueda de los archaî, de los principios. La posibilidad del málista eidénai es para Heidegger de importancia central en la medida en que expresa la concepción aristotélica del ser humano.

El movimiento de la zoé encuentra su mejor expresión en la caracterización del ser humano como zôon lógon échon ${ }^{25}$ o como aparece en el primer libro de la Ética Nicomáquea: praktiké tis toû lógon échontos ${ }^{26}$. El málista eidénai o "más de la visión” que intrínsecamente pertenece a la esencia del ser humano es posible mediante el lógos. Por ello Heidegger escribe en 1922 que "el griego ve al lógos como una determinada forma de la kínesis".

La lectura heideggeriana de Aristóteles centra su atención en los dos modos fundamentales de tener lógos a lo largo de la multiplicidad de

nexo en el cual se hallan los modos nombrados del trato: el movimiento de la vida, el ser de la vida." Cf. Heidegger: GA 62, p. 116 y 119.

${ }^{23}$ Met., I 1, 980a21. Para la versión castellana: Aristóteles, Metafísica, introducción, traducción y notas de Tomás Calvo, Madrid: Gredos 1994. En el semestre estival de 1922 Heidegger traduce este pasaje de la siguiente forma: "La exigencia de la vida en el ver (la absorción en lo visible) es algo que constituye el ser-cómo (modo ontológico de temporación del ser) del hombre", HeIDEgGER: GA 62, p. 17. Tres años después ofrece otra traducción: "En el ser del hombre reside en esencia el cuidado del ver", Martin Heidegger: Prolegómenos para una historia del concepto del tiempo, trad. Jaime Aspiunza, Madrid: Alianza 2006, p. 344 (alemán: Prolegomena zur Geschichte des Zeitbegriffs GA 20, Frankfurt am Main: Klostermann 1994, p. 380).

${ }^{24}$ Para lo que sigue me apoyo también en la lección que Heidegger sostuvo en Marburgo durante el semestre invernal de 1924/25: Platon: Sophistes, GA 19, Frankfurt

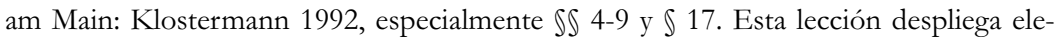
mentos ya vistos desde sus primeras interpretaciones en Friburgo. Cf. al respecto Walter BRÖCKER: Aristoteles, Frankfurt am Main: Klostermann 1935, especialmente \$S 1-3.

${ }^{25}$ De An., II 2, 432a30. Versión castellana: Aristóteles: Acerca del alma, introducción traducción y notas de Tomás Calvo, Madrid: Gredos 2000.

26 EN 1098a3. Versión castellana: ARISTÓTELES: Ética Nicomáquea, traducción y notas de Julio Pallí, Madrid: Gredos 1985. 
comportamientos que conforman la vida. Por un lado encontramos el movimiento que constata, nombrado por Aristóteles epistemonikón, es decir, la posibilidad científica en la que se lleva a cabo el lógos. Heidegger ubicará ahí el desarrollo del saber teorético ${ }^{27}$. Por otro lado está el movimiento que considera, el logistikón. Heidegger se referirá a éste como el desarrollo del deliberar. La parte constatadora se relaciona con los entes cuyos principios, archaí, no pueden ser de otra manera; mientras que la parte consideradora se relaciona con entes cuyos principios sí pueden ser de otra manera, ya que como señala Aristóteles "nadie delibera sobre lo que no puede ser de otra manera” (EN 1139a13).

Por lo cual hay diversos modos del aletheúein: por un lado son descubiertos los archaí que no pueden ser de otra forma; por otro lado, los que sí pueden ser de otra manera. Como modos del aletheúein que pertenecen a la parte constatadora encontramos la epistéme y la sophía. Mientras que la téchne y la phrónesis más bien corresponden a la parte consideradora. El noûs, por su lado, se muestra como lo posibilitador de los principios. Por ello Heidegger anota en el semestre invernal de 1924/25 que "los cuatro modos del aletheúein se dan en el noeîn; ellos son diversos modos de llevarse a cabo el noeîn" 28 .

Si la epistéme y la sophía constatan los principios que no pueden ser de otra manera, entonces se perfila el objeto de tal modo de darse la verdad: lo eterno. El objeto en este modo del lógon échon es el aídion, lo eterno. Saber en sentido estrecho significa saber de aquello que no puede ser de otra manera, saber de lo eterno (cf. EN 1139b23). Por ello aquí el "ver más", el málista eidénai, se dirige a los archaí de lo que no puede ser de otra forma, a lo eterno. La epistéme constituye el primer modo de saber al constatar lo que es. Pero tiene en sí la posibilidad de ser enseñable (didakté) y aprendible (mathetón) (EN 1139b25). Y precisamente estas posibilidades la constituyen como un comportamiento transmisible, como una enseñanza (didaskalía), la cual no requiere de un desencubrir propio de los principios. Ella parte de lo ya conocido, de lo proginoskoménon: presupone una base. En este sentido, la epistéme

\footnotetext{
${ }^{27}$ Cf. Heidegger: GA 19, p. 28. Además Bröcker: Aristoteles, p. 33.

${ }^{28}$ Heidegger: GA 19, p. 28.
} 
no es la beltíste héxis (EN 1139a15), la mejor disposición, dentro del epsitemonikón, ya que no puede abrir propiamente el arché del epistemonikón. El movimiento desencubridor de los principios se muestra limitado en la epistéme. Por ello debe darse otro movimiento que pueda abrir los archaí en el aídion. Y esto es obra de la sophía.

La sophía es pensada como la mejor disposición en el marco de lo que no puede ser de otra manera, precisamente porque en ese movimiento abre los archaí del epistemonikón mismo. Así, la mayor posibilidad, constatadora, científica, le corresponde al sabio.

Ahora bien, en el ámbito deliberador, logistikón, encontramos dos modos del aletheúein: la téchne y la phrónesis. La téchne sabemos que concreta el saber de la poíesis, del producir. Sin embargo, le corresponde a ésta el hecho de que su obra se halle fuera de ella misma, así el poietón o érgon que produce en tanto obra concluida ya no es objeto de la téchne, sino que ahora se halla junto al producir mismo. Ésta sería entonces una disposición en la cual no se abren sus archaí, al escapárseles siempre e independizarse.

Por su parte la phrónesis es aquel saber del logistikón, es decir, de lo que puede ser de otra manera, en donde la práxis misma se constituye como el objeto del saber ${ }^{29}$. La phrónesis sería así la posibilidad de desocultar la vida en tanto praktón.

Heidegger verá en la phrónesis la posibilidad de tematizar un saber que no se circunscribe al ámbito teorético, sino que abre caminos en torno a lo planteado ya por él como esfera preteorética. Será quizás por ello que en su curso de 1922 traduzca phrónesis como la "circunspección propia de la solicitud" [fürsorgliches Sichumsehen (Umsicht)] ${ }^{30}$.

El término 'circunspección' ya lo hemos anticipado al hablar de mundo circundante o vivencia del entorno. La vivencia del mundo circundante o entorno es el modo como inmediata y generalmente vivimos. En nuestra cotidianidad entornada, las vivencias del mundo circundante son aprehendidas mediante el comprender. Por ello Heidegger ve en el comprender el primer peldaño del método fenomenológico. El ver ate-

\footnotetext{
${ }^{29}$ Cf. EN VI 5, 1140a24-ss.; 8-9, 1141b33-ss.; Eth. Eud., VIII 1, 1246 b36.

${ }^{30}$ HeidegGer: GA 62, pp. 376-s.
} 
mático que ocurre en la vivencia del entorno es aquello que Heidegger nombra desde 1919 "intuición comprendedora". Y ya que aquello visto o intuido es lo entornado, entonces este ver debe entenderse como ver en torno, como circunspección [Umsicht]. El comprender no refiere pues a ningún ver dirigido de modo objetivante, sino a un ver circunspectivo en donde las cosas de entrada aparecen como entornadas, como significativas. Pero en tanto que este ver es un ver atemático ¿cómo puede entenderse la posibilidad de una tematización?

Como ya indicamos, los modos del aletheúein aristotélicos son para Heidegger modos de acceso, es decir, modos de hacer expreso. Ya que la vida debe mantener sus caracteres fundamentales circunspectivos de mundo circundante o entorno, para no convertirse en una simple objetivación, la tematización ejecutante de la vida recibe sus impulsos a partir de las aproximaciones a la phrónesis aristotélica, en donde para Heidegger lo enfático recae en ver a ésta como un modo de acceso a la vida misma, como un modo de desocultamiento, como un modo del aletheúein.

El "ver más", málista eidénai, que ocurre en la phrónesis no es ningún conocimiento "objetivo", sino un movimiento que se halla en la misma vida y muestra la forma como ésta puede ser aprehendida. El phrónimos no es el hombre que observa objetivamente, sino el que puede deliberar adecuadamente: kalôs bouleúsasthai (EN 1140a26). Y esto lo hace en relación con su propia vida. El objeto de la phrónesis es pues el bouletikós mismo. Por ello en el bouleúesthai del phrónimos es vista o abierta la zoé misma. El télos del phrónimos no es ningún elemento traído desde afuera, sino que es he eupraxía, el actuar adecuado (EN 1140b6-ss). Por ello Heidegger escribe en el semestre invernal de 1924/25: "Más bien en la phrónesis el objeto de la deliberación es la zoé misma; el télos tiene el mismo carácter de ser que la phrónesis" y un poco más abajo: "En la phrónesis el praktón tiene el mismo carácter de ser que el aletheúeien mismo"31.

Al llegar a este punto podemos retomar lo ya desarrollado. Si la phrónesis es un modo determinado del descubrir y tiene el mismo ca-

${ }^{31}$ Heidegger: GA 19, p. 49. 
rácter que lo descubierto, es decir, el mismo carácter de ser que la vida, entonces aquí se puede ver con mayor claridad el impulso metódico aristotélico para la fenomenología hermenéutica de Heidegger. Dicho de otra forma: si para Aristóteles la phrónesis es un modo adecuado para el descubrimiento de la vida, y pertenece a ésta, entonces la interpretación tematizadora de la fenomenología hermenéutica se apega a esta posibilidad no teorética en la apertura de la vida. Por eso Heidegger escribirá a Jaspers en 1922 que "hay objetos, que no se tiene, sino que se 'es'; mas aún, el qué de estos objetos descansa en 'que son" 32 .

Este modo preteorético de acceso a la vida será el hilo conductor que tome Heidegger en su camino hacia Ser y tiempo. Por eso la interpretación no será para Heidegger un elemento ajeno a la vida misma, sino el desarrollo hermenéutico de lo comprendido en la vivencia del entorno $^{33}$. De ese modo, el señalamiento posterior de Ser y tiempo que indica que "en la interpretación el comprender no es algo diferente, sino él mismo" "34 reluce de modo fehaciente en la apropiación ontológica que Heidegger lleva a cabo en torno a la phrónesis aristotélica, ya que si el desarrollo del comprender tiene el mismo carácter de la vida, entonces el télos de tal desarrollo no es otro sino la posibilidad de hacer expresa la vida misma, es decir, la posibilidad de una fenomenología hermenéutica de la facticidad de la vida.

${ }^{32}$ Martin Heidegger y Karl Jaspers: Correspondencia (1920-1963), Madrid: Síntesis 2003, p. 24 (alemán: Briefwechsel 1920-1963, München: Piper 1990, p. 26). Se trata de la carta del 27 de junio de 1922.

${ }^{33}$ Ya otros trabajos han destacado cómo es que la interpretación se convierte en el desarrollo hermenéutico de lo comprendido. Baste por el momento remitir a algunos de ellos, ya que tratar esto aquí desbordaría los objetivos del presente escrito. Cf. Ángel Xolocotzi: Fenomenología de la vida fáctica. Heidegger y su camino a Ser y tiempo, México: Plaza y Valdés 2004; Ramón RODRÍGUEZ: La transformación hermenéutica de la fenomenología, Madrid: Tecnos 1997; Modesto BERCiAnO: La revolución filosófica de Martin Heidegger, Madrid: Biblioteca Nueva 2001; Joseph María BECH: De Husserl a Heidegger. La transformación del pensamiento fenomenológico, Barcelona: Universitat de Barcelona 2001.

${ }^{34}$ Martin Heidegger: Ser y tiempo, trad. Jorge Eduardo Rivera, Santiago de Chile: Universitaria 1997, p. 148 (alemán: Sein und Zeit, Tübingen: Niemeyer 1927, p. 124). 


\section{Conclusión}

La literatura secundaria sigue mostrando que el acercamiento a Heidegger difícilmente se da sine ira et studio. Las diversas reacciones no se limitan al cuestionamiento en torno a las relaciones entre vida y obra, sino al manejo que Heidegger llevó a cabo de la tradición filosófica occidental. Es incuestionable la magnitud de su lectura de los filósofos de Occidente, comparable quizás sólo a lo que hizo Hegel; sin embargo, muchos cuestionan la fuerza interpretativa con la que se acercó a los autores "violentando" así los cánones hermenéuticos.

Más allá de la ortodoxia, y a pesar de la "violencia interpretativa" que se le atribuye a Heidegger, la apertura de la tradición filosófica de modo radical ha abierto caminos que ahora ya son un lugar común. Baste recordar la novedosa lectura ontológica que nuestro autor hace de Kant en contra de las lecturas epistemológicas que se veían como la única posibilidad. Asimismo, la publicación de sus Nietzsche en los años 60 recordaron que éste podía ser leído como filósofo y no sólo como esteta.

Con Aristóteles ocurre también una apertura radical al releerlo desde las pretensiones ateoréticas que lo movían por lo menos a partir de 1919. De esa forma, como vimos, Heidegger ve en Aristóteles aquel gigante con el cual medir sus fuerzas y obtener una base firme para su acceso a la vida fáctica. Fue entonces el estagirita el que determinó en gran medida la posterior obra heideggeriana. Sin temor a exagerar podemos confirmar, como ya lo había hecho Franco Volpi hace algunas décadas, que sin Aristóteles Heidegger no podía haber llegado a ser el filósofo determinante que ha sido.

En las últimas décadas los estudiosos de la obra de Heidegger han destacado dos direcciones que toma la rehabilitación heideggeriana de la phrónesis aristotélica: como filosofía práctica y como ámbito preteorético que decanta en una apropiación ontológica. En el primer caso encontramos su escuela: Gadamer, Arendt, Ritter, Jonas, Strauss. En el segundo caso, en el cual nos hemos centrado aquí, queda sólo él. En ambas perspectivas se cuestiona tajantemente la hegemonía teorética del filosofar occidental y se abren perspectivas para el pensar. 
Así, actualmente, a varias décadas de la histórica interpretación heideggeriana de Aristóteles, de la muerte de Heidegger, del descubrimiento volpiano del aristotelismo de Heidegger, podemos evaluar que la interpretación heideggeriana de Aristóteles ha sido un suceso determinante para la filosofía del siglo XX y de esa forma también podemos entender mejor aquella célebre valoración de Leo Strauss en torno a su experiencia con Heidegger en 1922, y quizás también suscribirla:

No había visto nunca antes tanta seriedad, profundidad y concentración en la interpretación de los textos filosóficos. Había escuchado la interpretación que Heidegger daba de ciertos pasajes de Aristóteles y algún tiempo después escuché a Werner Jaeger en Berlín interpretar los mismos textos: la compasión me exige limitar mi comparación a la observación de que no había comparación ${ }^{35}$.

${ }^{35}$ Leo STRAuss: "Introducción al existencialismo de Heidegger", Sobre Heidegger. Cinco voces judías, Introducción de Franco Volpi, Buenos Aires: Manantial 2008, p. 42 (inglés: The Rebirth of Classical Political Rationalism, Chicago 1989, p. 27-s.). 
